around roo $\mathrm{kg}$. Complete data were recorded in 108 pigs from 28 litters sired by 8 Landrace boars (XL group) and 122 pigs from 30 litters sired by 7 Pietrain boars (XP group). $A$ least-squares analysis was performed to obtain an estimate of the "breed of boar" effect for 24 traits. XL pigs prevailed over XP pigs in an average claily gain ( $82 \mathrm{I} v 5787 \mathrm{~g} ; \mathrm{P}<0.0 \mathrm{I}$ ) but food conversion ratio, measured between 30 and $90 \mathrm{~kg}$, was quite similar in the two groups ( $3.15 \mathrm{vs} 3.16$ ); average daily food consumption was lower by 6 p. Ion $(\mathrm{P}<0,0 \mathrm{I})$ in Pietrain crosses. A highly significant difference (XL-XP) was found for dressing out percentage $(-\mathbf{0 . 7} \pm 0.2)$ carcass length $(+50 \pm 4 \mathrm{~mm})$, weight of ham $(-0.12 \pm 0.04 \mathrm{~kg})$, weight of loin $(-0.30+0.08 \mathrm{~kg})$. No difference between breeding groups was found in weight of backfat and average backfat thickness, but weight of leaf was much lower (- I5 p. roo) in XP pigs. With respect to meat quality, assessed 24 hours post mortem, a general superiority of XL pigs as compared to XP pigs was evidenced, especially for water binding capacity and color of two muscles; XL pigs presented an advantage in $\mathrm{pH}$ of muscle L. dorsi ( 5.95 vs $5.39, \mathrm{P}<0.05$ ) but there was no significant difference in $\mathrm{p} \mathrm{H}$ of 3 muscles of the ham. Despite a higher fattening cost $\left(+{ }_{4} \mathrm{~F} / \mathrm{pig}\right)$ and because of a greater commercial value of the carcass $(+8 \mathrm{~F} / \mathrm{pig})$, the 3 -way cross with Pietrain showed an advantage of about ${ }_{4} \mathrm{~F}$ per pig as compared to the back cross with French Landrace in overall economic merit. The genetic signification of these differences between crossbreds is discussed in relation to an earlier comparison between pure Landrace and Pietrain gilts.

\title{
The improvement of pig meat quality through selection
}

\author{
L. OLLIVIER and D. POTIER
}

Station de Génétique quantitative et appliquée, I. N. R. A., C. N.R. Z., 78350 Jouy en Josas

This paper discusses the choice and combination of selection criteria in order to improve meat quality in pigs. Several measurements on the live animal have been studied recently and have been shown to be more or less closely associated with meat quality. From a genetic point of view, a high selection intensity on such criteria can make for their low precision. The efficiency of individual sclection depends on the product $r h^{\prime}, h^{\prime}$ being the heritability of a live measurement and $r$ being its genetic correlation with meat quality. Thus, with equal selection intensities, $r h^{\prime}$ must at least be equal to $0.35 \mathrm{in}$ order that individual selection be as efficient as a 2 sib-test (on meat quality with 0.30 as heritability and 0.25 as phenotypic correlation between full-sibs). But, when selection is 6 times as intense on individual performance as with sib or combined selection (which is the present situation in Francc), the lower limit of $r h^{\prime}$, which makes individual selection as efficient as the other 2 methods, becomes 0.06 .

When the aim is simultaneously to improve feed efficiency, carcass and meat quality, the relative weight of each of these characters must be established. In French conditions of 1974, a standard-deviation of meat quality is worth ro F., through its incidence on fattening and transport losses and on the yield of industrial transformations. For fattening cost and carcass valuc, the standard-deviations are both worth around $20 \mathrm{~F}$. With those economic weights, selection indices for combined testing (with 2 sibs) are 20 p. Ioo more efficient than individual selection indices, in terms of correlation between index and aggregate genetic valuc. The relative values of these correlations are practically independant of the genetic parameters of the live measurement predicting 
meat quality. Such measurements can thus be ignored without great loss in total genetic progress. This is because of a balance between progress in meat quantity and in meat quality. But, as expected, the genetic parameter relative to the live measurement for meat quality are not irrelevant when genetic progress in meat quality is being considered. The first parameter of importance is the correlation $r^{\prime}$ (usually negative) between meat quality, as estimated on the live animal, and meat quantity in the carcass. When $r^{\prime}$ is low, the product $r h^{\prime}$, as defined above, must be higher than 0.25 in order to gain in meat quality. When $r^{\prime}$ is highly negative, this threshold becomes much higher, and, with usual values for $r$ and $h^{\prime}$, the addition of a live criterion for meat quality in the individual index brings a faster genetic decline in meat quality, unless use is made of restricted selection indices, which means a lesser global efficiency. 